TRANSACTIONS OF THE

AMERICAN MATHEMATICAL SOCIETY

Volume 351, Number 8, Pages 3217-3228

S 0002-9947(99)02027-9

Article electronically published on April 20, 1999

\title{
A THEOREM ON ZETA FUNCTIONS ASSOCIATED WITH POLYNOMIALS
}

\author{
MINKING EIE AND KWANG-WU CHEN
}

Abstract. Let $\beta=\left(\beta_{1}, \ldots, \beta_{r}\right)$ be an $r$-tuple of non-negative integers and $P_{j}(X)(j=1,2, \ldots, n)$ be polynomials in $\mathbb{R}\left[X_{1}, \ldots, X_{r}\right]$ such that $P_{j}(n)>0$ for all $n \in \mathbb{N}^{r}$ and the series

$$
\sum_{n \in \mathbb{N}^{r}} P_{j}(n)^{-s}
$$

is absolutely convergent for $\operatorname{Re} s>\sigma_{j}>0$. We consider the zeta functions

$$
Z\left(P_{j}, \beta, s\right)=\sum_{n \in \mathbb{N}^{r}} n^{\beta} P_{j}(n)^{-s}, \quad \operatorname{Re} s>|\beta|+\sigma_{j}, \quad 1 \leq j \leq n .
$$

All these zeta functions $Z\left(\prod_{j=1}^{n} P_{j}, \beta, s\right)$ and $Z\left(P_{j}, \beta, s\right) \quad(j=1,2, \ldots, n)$ are analytic functions of $s$ when $\operatorname{Re} s$ is sufficiently large and they have meromorphic analytic continuations in the whole complex plane.

In this paper we shall prove that

$$
Z\left(\prod_{j=1}^{n} P_{j}, \beta, 0\right)=\frac{1}{n} \sum_{j=1}^{n} Z\left(P_{j}, \beta, 0\right) .
$$

As an immediate application, we use it to evaluate the special values of zeta functions associated with products of linear forms as considered by Shintani and the first author.

\section{INTRODUCTION AND NOTATION}

As usual, we let $\mathbb{R}$ and $\mathbb{C}$ be the field of real numbers and the field of complex numbers, respectively. $\mathbb{N}$ denotes the set of positive integers.

Let $P_{j}\left(X_{1}, \ldots, X_{r}\right)(j=1, \ldots, n)$ be polynomials of $r$ variables such that $P_{j}(n)$ $>0$ for all $n \in \mathbb{N}^{r}$ and the series

$$
\sum_{n \in \mathbb{N}^{r}} P_{j}(n)^{-s}=\sum_{n_{1}=1}^{\infty} \ldots \sum_{n_{r}=1}^{\infty} P\left(n_{1}, \ldots, n_{r}\right)^{-s}
$$

is absolutely convergent for $\operatorname{Re} s>\sigma_{j}>0$. Let $\beta=\left(\beta_{1}, \ldots, \beta_{r}\right)$ be an $r$-tuple of non-negative integers. Consider the zeta function $Z\left(P_{j}, \beta, s\right)$ associated with $P_{j}$

Received by the editors August 11, 1995 and, in revised form, February 4, 1997.

1991 Mathematics Subject Classification. Primary 11M06.

This work was supported by Department of Mathematics, National Chung Cheng University and National Science Foundation of Taiwan, Republic of China.

(C)1999 American Mathematical Society 
defined as

$$
\begin{aligned}
Z\left(P_{j}, \beta, s\right) & =\sum_{n \in \mathbb{N}^{r}} n^{\beta} P_{j}(n)^{-s} \\
& =\sum_{n_{1}=1}^{\infty} \ldots \sum_{n_{r}=1}^{\infty} n_{1}^{\beta_{1}} \ldots n_{r}^{\beta_{r}} P_{j}\left(n_{1}, \ldots, n_{r}\right)^{-s}, \quad \operatorname{Re} s>\sigma_{j}+|\beta|,
\end{aligned}
$$

where $|\beta|=\beta_{1}+\ldots+\beta_{r}$.

To see the analytic continuations of this kind of zeta functions, we need

Proposition 1 (Zagier [11]). Suppose that $\varphi(s)=\sum_{\lambda>0} \alpha_{\lambda} \lambda^{-s}$ ( $\lambda$ ranges over a sequence of positive numbers tending $+\infty$ ) is a Dirichlet series converging for sufficiently large Re $s$, then $f(t)=\sum_{\lambda>0} a_{\lambda} e^{-\lambda t}$ is the corresponding exponential series. If at $t=0, f(t)$ has the asymptotic expansion $\sum_{n \geq n_{0}} C_{n} t^{n / p}$ where $n_{0}$ is an integer and $p$ is a fixed positive integer. Then

(1) $\varphi(s)$ has a meromorphic continuation in the whole complex plane.

(2) $\varphi(s)$ has a possible simple pole at $s=-n / p$, where $n$ is not a multiple of $p$ if $n>0$, with residue $C_{n} / \Gamma(-n / p)$ and no other poles.

(3) $\varphi(0)=C_{0}$.

For a given polynomial $P(X) \in \mathbb{R}\left[X_{1}, \ldots, X_{r}\right]$ such that $P(n)>0$ for all $n \in \mathbb{N}^{r}$, the series

$$
\sum_{n \in \mathbb{N}^{r}} P_{j}(n)^{-s}
$$

is absolutely convergent for $\operatorname{Re} s>\sigma$. Suppose that

$$
P(X)=\sum_{|\alpha|=0}^{p} C_{\alpha} X^{\alpha}, \quad p=\operatorname{deg} P(X) ;
$$

where $|\alpha|=\alpha_{1}+\cdots+\alpha_{r}$ is the degree of $X^{\alpha}=X_{1}^{\alpha_{1}} \cdots X_{r}^{\alpha_{r}}$. Let

$$
Q(X, t)=\sum_{|\alpha|=0}^{p} C_{\alpha} X^{\alpha} t^{p-|\alpha|} .
$$

Then $Q(X, t)$ is a homogeneous polynomial of degree $p$ such that

$$
Q(X t, t)=t^{p} Q(X, 1)=t^{p} P(X) .
$$

As in [11], by applying the classical Euler-Maclaurin summation formula repeatedly to the function

$$
\begin{aligned}
g(t) & =\sum_{n \in \mathbb{N}^{r}}(n t)^{\beta} e^{-Q(n t, t)} \\
& =\sum_{n_{1}=1}^{\infty} \cdots \sum_{n_{r}=1}^{\infty}\left(n_{1} t\right)^{\beta_{1}} \ldots\left(n_{r} t\right)^{\beta_{r}} e^{-Q\left(n_{1} t, \ldots, n_{r} t, t\right)}, \quad t>0,
\end{aligned}
$$

we get that $g(t)$ has an asymptotic expansion of the form

$$
\sum_{n \geq m_{0}} a_{n} t^{n}
$$


as $t$ approaches 0 . It follows that

$$
f(t)=\sum_{n \in \mathbb{N}^{r}} n^{\beta} \exp \{-P(n) t\}
$$

has an asymptotic expansion of the form

$$
\sum_{n \geq n_{0}-|\beta|} b_{n} t^{n / p}
$$

as $t$ approaches 0 . By Proposition $1, Z(P, \beta, s)$ has its meromorphic continuation in the whole complex $s$-plane. Therefore both the zeta functions $Z\left(\prod_{j=1}^{n} P_{j}, \beta, s\right)$ and $Z\left(P_{j}, \beta, s\right)(j=1, \ldots, n)$ are analytic functions of $s$ in some half planes and have meromorphic analytic continuations in the whole complex plane.

Here we shall prove a relation concerning the special values at $s=0$ of these zeta functions associated with polynomials.

Theorem. Suppose that $P_{j}(j=1, \ldots, n)$ are polynomials of $r$ variables with nonnegative coefficients such that $P(n)>0$ for all $n \in \mathbb{N}^{r}$ and the series

$$
Z\left(P_{j}, \beta ; s\right)=\sum_{n \in \mathbb{N}^{r}} n^{\beta} P_{j}(n)^{-s}
$$

is absolutely convergent for Re $s>\sigma_{j}>0$. Then $Z\left(P_{j}, \beta ; s\right)(j=1, \ldots, n)$ as well as $Z\left(\prod_{j=1}^{n} P_{j}, \beta ; s\right)$ have their analytic continuations. Furthermore, one has

$$
Z\left(\prod_{j=1}^{n} P_{j}, \beta ; 0\right)=\frac{1}{n} \sum_{j=1}^{n} Z\left(P_{j}, \beta ; 0\right) .
$$

Let $P$ be a polynomial having the same properties as $P_{j}$ mentioned above. From the relation

$$
Z(P, \beta, s-m)=\sum_{n \in \mathbb{N}^{r}} n^{\beta} P^{m}(n) P(n)^{-s}, \quad \operatorname{Re} s>m+\sigma+|\beta|,
$$

we can express the special value $Z(P, \beta,-m)$ in terms of a linear combination of $Z(P, \alpha, 0)$ for various $\alpha$. Indeed if

$$
X^{\beta} P^{m}(X)=\sum_{|\alpha|=|\beta|}^{m p+|\beta|} C_{\alpha} X^{\alpha}, p=\operatorname{deg} P(X),
$$

then

$$
Z(P, \beta,-m)=\sum_{|\alpha|=|\beta|}^{m p+|\beta|} C_{\alpha} Z(P, \alpha, 0) .
$$

Thus, such a relation is useful to compute the special values at non-positive integers of zeta functions associated with polynomials. As applications, we shall give the formula for special values of $Z\left(\prod_{j=1}^{n} L_{j}, \beta, s\right)$ with $L_{j}$ a linear form

$$
L_{j}(X)=a_{j 1} X_{1}+\ldots+a_{j r} X_{r}+\delta_{j}, \quad \operatorname{Re} a_{j i}>0,
$$

as considered by the first author in [6], and the zeta function $\zeta(A, x, s)$ considered by Shintani [9], defined by

$$
\zeta(A, x, s)=\sum_{n_{1}=0}^{\infty} \ldots \sum_{n_{r}=0}^{\infty} \prod_{j=1}^{n}\left(a_{j 1}\left(n_{1}+x_{1}\right)+\ldots+a_{j r}\left(n_{r}+x_{r}\right)\right)^{-s} .
$$




\section{THE PROOF OF THE THEOREM}

By Proposition 1 , computing $Z(P, \beta, 0)$ is equivalent to computing the constant term in the asymptotic expansion at $t=0$ of the function

$$
f(P, t)=\sum_{n \in \mathbb{N}^{r}} n^{\beta} \exp \{-P(n) t\} .
$$

To prove our theorem, we have to consider $f\left(\prod_{j=1}^{n} P_{j}, t\right)$ and $f\left(P_{j}, t\right)(j=1,2, \ldots, n)$ simultaneously. First we need the following lemmas concerning integrations over the standard simplex of $\mathbb{R}^{n}$.

Let $E^{n}$ be the standard simplex in $\mathbb{R}^{n}$ defined by

$$
E^{n}=\left\{u=\left(u_{1}, \ldots, u_{n}\right) \in \mathbb{R}^{n} \mid 0 \leq u_{i} \leq 1, \sum_{i=1}^{n} u_{i}=1\right\} .
$$

$d U=d u_{1} \cdots d u_{n-1}$ is the standard Euclidean measure on $E^{n}$. Denote by $V_{1}=$ $(1,0, \ldots, 0), V_{2}=(0,1,0, \ldots, 0), \ldots, V_{n}=(0, \ldots, 0,1)$ the vertexes of $E_{n}$. For any positive number $h, 0<h<1$, we let

$$
E_{j}^{n}(h)=\left\{u=\left(u_{1}, \ldots, u_{n}\right) \in E^{n} \mid 1-h \leq u_{j} \leq 1\right\} .
$$

Lemma 1. Let $g$ be a continuous function on $[0, \infty)$ and let $\alpha_{1}, \cdots, \alpha_{n-1}$ be nonnegative integers. Then for Res $>0$,

$$
\begin{aligned}
\int_{E_{n}^{n}(h)} u_{1}^{s+\alpha_{1}-1} \cdots u_{n-1}^{s+\alpha_{n-1}-1} g\left(u_{1}+\cdots+u_{n-1}\right) d u_{1} \cdots d u_{n-1} \\
=\frac{\Gamma\left(s+\alpha_{1}\right) \cdots \Gamma\left(s+\alpha_{n-1}\right)}{\Gamma\left((n-1) s+\alpha_{1}+\cdots+\alpha_{n-1}\right)} \int_{0}^{h} t^{(n-1) s+\alpha_{1}+\cdots+\alpha_{n-1}-1} g(t) d t
\end{aligned}
$$

Proof. With the change of variables

$$
\left\{\begin{array}{l}
t=u_{1}+\cdots+u_{n-1} \\
v_{j}=\frac{u_{j}}{t}, \quad 1 \leq j \leq n-1
\end{array}\right.
$$

Then $v=\left(v_{1}, \cdots, v_{n-1}\right)$ lies in the standard simplex $E^{n-1}$ and the integral is transformed into

$$
\int_{E^{n-1}} v_{1}^{s+\alpha_{1}-1} \cdots v_{n-1}^{s+\alpha_{n-1}-1} d V \int_{0}^{h} t^{(n-1) s+|\alpha|-1} g(t) d t
$$

Our assertion then follows from a theorem concerning $\beta$-functions of several variables [8].

Lemma 2. Suppose that $f(u)$ is an analytic function on the simplex $E^{n}$ and

$$
G(s)=\frac{1}{\Gamma(s)^{n-1}} \int_{E^{n}}\left(u_{1} \ldots u_{n}\right)^{s-1} f(u) d U, \quad \text { Res }>0 .
$$

Then $G(s)$ has its analytic continuation for Re $s>-1$ and $G(0)=\sum_{j=1}^{n} f\left(V_{j}\right)$.

Proof. Consider the integral around $V_{j}$ defined by

$$
G_{j}(s)=\frac{1}{\Gamma(s)^{n-1}} \int_{E_{j}^{n}(h)}\left(u_{1} \ldots u_{n}\right)^{s-1} f(u) d U .
$$


Suppose that on $E_{j}^{n}(h), f$ has the expansion

$$
f(u)=f\left(V_{j}\right)+\sum_{|\alpha| \geq 1} C_{\alpha} u_{1}^{\alpha_{1}} \cdots u_{j-1}^{\alpha_{j-1}} u_{j+1}^{\alpha_{j+1}} \cdots u_{n}^{\alpha_{n}} .
$$

Now consider the case $j=n$. From Lemma 1 and a term by term integration, for $\operatorname{Re} s>0$,

$$
\begin{aligned}
G_{n}(s)= & f\left(V_{n}\right) \frac{1}{\Gamma((n-1) s)} \int_{0}^{h} t^{(n-1) s-1}(1-t)^{s-1} d t \\
& +\sum_{|\alpha| \geq 1} C_{\alpha} \frac{\Gamma\left(s+\alpha_{1}\right) \cdots \Gamma\left(s+\alpha_{n-1}\right)}{\Gamma(s)^{n-1} \Gamma((n-1) s+|\alpha|)} \int_{0}^{h} t^{(n-1) s+|\alpha|-1}(1-t)^{s-1} d t
\end{aligned}
$$

In the above expression for $G_{n}(s)$, write the first term as

$$
\begin{aligned}
& f\left(V_{n}\right) \frac{1}{\Gamma((n-1) s)}\left(\int_{0}^{h} t^{(n-1) s-1} d t+\int_{0}^{h} t^{(n-1) s-1}\left[(1-t)^{s-1}-1\right] d t\right) \\
& =f\left(V_{n}\right) \frac{h^{(n-1) s}}{\Gamma((n-1) s+1)}+f\left(V_{n}\right) \frac{1}{\Gamma((n-1) s)} \int_{0}^{h} t^{(n-1) s-1}\left[(1-t)^{s-1}-1\right] d t .
\end{aligned}
$$

Hence $G_{n}(s)$ has analytic continuation for $\operatorname{Re} s>-1$ and its special value at $s=0$ is given by $f\left(V_{n}\right)$.

For the remaining terms, the integrals

$$
\int_{0}^{h} t^{(n-1) s+|\alpha|-1}(1-t)^{s-1} d t, \quad|\alpha| \geq 1
$$

are convergent for $s=0$ and the gamma functions

$$
\frac{\Gamma\left(s+\alpha_{1}\right) \cdots \Gamma\left(s+\alpha_{n-1}\right)}{\Gamma(s)^{n-1} \Gamma((n-1) s+|\alpha|)}, \quad|\alpha| \geq 1,
$$

are analytic at $s=0$ and vanish there. Thus we have

$$
G_{n}(0)=f\left(V_{n}\right)
$$

In general, we have

$$
G_{j}(0)=f\left(V_{j}\right) .
$$

Note that $H(s)=G(s)-\sum_{j=1}^{n} G_{j}(s)$ vanishes at $s=0$ owing to the zero of order $n-1$ from the factor $\Gamma(s)^{1-n}$. Thus

$$
G(0)=\sum_{j=1}^{n} G_{j}(0)=\sum_{j=1}^{n} f\left(V_{j}\right)
$$

Proof of the theorem. The existence of analytic continuations follows from a standard argument as mentioned before (see also [3]). Here we prove the equality. For Re $s>\max \left(\sigma_{1}, \ldots, \sigma_{n}\right)$, we have

$$
\begin{aligned}
\prod_{j=1}^{n} P_{j}(n)^{-s}[\Gamma(s)]^{n} & =\int_{0}^{\infty} \cdots \int_{0}^{\infty}\left(t_{1} \cdots t_{n}\right)^{s-1} \exp \left\{-\sum_{j=1}^{n} P_{j}(n) t_{j}\right\} d t_{1} \cdots d t_{n} \\
& =\int_{0}^{\infty} t^{n s-1} d t \int_{E^{n}}\left(u_{1} \cdots u_{n}\right)^{s-1} \exp \left\{-\sum_{j=1}^{n} P_{j}(n) u_{j} t\right\} d U
\end{aligned}
$$


It follows for sufficiently large Re $s$,

$$
Z\left(\prod_{j=1}^{n} P_{j}, \beta ; s\right) \Gamma(s)=\int_{0}^{\infty} t^{n s-1} d t \frac{1}{\Gamma(s)^{n-1}} \int_{E^{n}}\left(u_{1} \cdots u_{n}\right)^{s-1} f(u, t) d U
$$

where $f(u, t)=\sum_{n \in \mathbb{N}^{r}} n^{\beta} \exp \left\{-\sum_{j=1}^{n} P_{j}(n) u_{j} t\right\}$. Set

$$
F(s, t)=\frac{1}{\Gamma(s)^{n-1}} \int_{E^{n}}\left(u_{1} \ldots u_{n}\right)^{s-1} f(u, t) d U .
$$

As a function of $s, F(s, t)$ is an analytic function of $s$ for $\operatorname{Re} s>0$. By the previous lemma, $F(s, t)$ has its analytic continuation for $\operatorname{Re} s>-1$. Furthermore,

$$
F(0, t)=\sum_{j=1}^{n} \sum_{n \in \mathbb{N}^{r}} n^{\beta} \exp \left\{-P_{j}(n) t\right\} .
$$

For any $\epsilon>0$, let $L(\epsilon)$ be the contour in a complex plane consisting of $[\epsilon,+\infty)$ twice in the opposite direction and the circle $|t|=\epsilon$ counterclockwise. Then we have

$$
\int_{L(\epsilon)} t^{n s-1} F(s, t) d t=\left(e^{2 \pi i n s}-1\right) \int_{\epsilon}^{\infty} t^{n s-1} F(s, t) d t+\int_{|t|=\epsilon} t^{n s-1} F(s, t) d t .
$$

For sufficiently large $\operatorname{Re} s$, the second integral approaches to zero as $\epsilon \rightarrow 0$. Consequently for sufficiently small $\epsilon>0$ and sufficiently large $\operatorname{Re} s$, one has

$$
Z\left(\prod_{j=1}^{n} P_{j}, \beta ; s\right) \Gamma(s)=\frac{1}{e^{2 \pi i n s}-1} \int_{L(\epsilon)} t^{n s-1} F(s, t) d t .
$$

With the well-known functional equation

$$
\Gamma(s) \Gamma(1-s)=\frac{\pi}{\sin \pi s}=\frac{2 \pi i e^{\pi i s}}{e^{2 \pi i s}-1},
$$

we rewrite the formula as

$$
Z\left(\prod_{j=1}^{n} P_{j}, \beta ; s\right)=\Gamma(1-s) e^{-\pi i s} \frac{e^{2 \pi i s}-1}{e^{2 \pi i n s}-1} \cdot \frac{1}{2 \pi i} \int_{L(\epsilon)} t^{n s-1} F(s, t) d t .
$$

Note that the above contour integral is absolutely convergent for all $s$, hence it gives the analytic continuation of $Z\left(\prod_{j=1}^{n} P_{j}, \beta ; s\right)$. When $s=0$, the integration along $[\epsilon,+\infty)$ twice in the opposite direction will be cancelled. Hence

$$
\begin{aligned}
Z\left(\prod_{j=1}^{n} P_{j}, \beta ; 0\right) & =\frac{1}{n} \cdot \frac{1}{2 \pi i} \int_{|t|=\epsilon} t^{-1} F(0, t) d t \\
& =\frac{1}{n} \times\{\text { the constant term in the } \\
& \text { asymptotic expansion of } F(0, t) \text { at } t=0\} .
\end{aligned}
$$

On the other hand, we have for $\operatorname{Re} s>\sigma_{j}+|\beta|$,

$$
Z\left(P_{j}, \beta ; s\right) \Gamma(s)=\int_{0}^{\infty} t^{s-1} \sum_{n \in \mathbb{N}^{r}} n^{\beta} \exp \left\{-P_{j}(n) t\right\} d t .
$$


Hence

$$
\begin{aligned}
& Z\left(P_{j}, \beta ; 0\right)=\text { the constant term in the asymptotic expansion of } \\
& \qquad \sum_{n \in \mathbb{N}^{r}} n^{\beta} \exp \left\{-P_{j}(n) t\right\} \text { at } t=0 .
\end{aligned}
$$

Thus our assertion follows.

Remark. The condition that $P_{j}(X)(j=1, \ldots, n)$ are polynomials of non-negative coefficients such that $P(n)>0$ for all $n \in \mathbb{N}^{r}$ can be relaxed. Indeed, Re $P_{j}(n)>$ $0(j=1, \ldots, n)$, along with some convergence condition on the series, is enough for our purpose.

\section{Applications}

Here we only mention two main applications of our theorem. Other applications to evaluate the special values of particular zeta functions associated with polynomials are also possible.

Let $P(X)$ be a product of $n$ linear forms in $r$ variables $X_{1}, \ldots, X_{r}$ as given by

$$
\begin{array}{r}
P\left(X_{1}, \ldots, X_{r}\right)=\prod_{j=1}^{n} L_{j}(X)=\prod_{j=1}^{n}\left(a_{j 1} X_{1}+\ldots+a_{j r} X_{r}+\delta_{j}\right) \\
\operatorname{Re} a_{j i}>0, \quad \operatorname{Re}\left(\delta_{j}+\sum_{i=1}^{r} a_{j i}\right)>0 .
\end{array}
$$

In [6], the first author considered the zeta function

$$
Z(P, \beta, s)=\sum_{n_{1}=1}^{\infty} \ldots \sum_{n_{r}=1}^{\infty} n_{1}^{\beta_{1}} \ldots n_{r}^{\beta_{r}} P\left(n_{1}, \ldots, n_{r}\right)^{-s}, \quad \operatorname{Re} s>\frac{r+|\beta|}{n},
$$

and he obtained an explicit expression of $Z(P, \beta,-m)$ in terms of polynomials in Bernoulli numbers defined by

$$
\frac{t}{e^{t}-1}=\sum_{n=0}^{\infty} \frac{B_{n} t^{n}}{n !}, \quad|t|<2 \pi .
$$

As mentioned before, $Z(P, \beta,-m)$ is a linear combination of $Z(P, \alpha, 0)$ for various $\alpha$. Also by our theorem, $Z(P, \alpha, 0)$ is the average of $Z\left(L_{j}, \alpha, 0\right), j=1, \ldots, n$. So it suffices to evaluate $Z\left(L_{j}, \alpha, 0\right)$ in order to compute $Z(P, \beta,-m)$.

Here we use the notation from [6] and let $J^{p}$ be the linear extension of $\mathbb{C}\left[X_{1}, \ldots, X_{p}\right]$ to $\mathbb{C}$ satisfying

$$
J^{p}\left[X_{1}^{\alpha_{1}} \ldots X_{p}^{\alpha_{p}}\right]=\prod_{i=1}^{p} \zeta\left(-\alpha_{i}\right)=\prod_{i=1}^{p} \frac{(-1)^{\alpha_{i}} B_{\alpha_{i}+1}}{\alpha_{i}+1} .
$$

Proposition 2. Let $L(X)=a_{1} x_{1}+\ldots+a_{r} x_{r}+\delta$ be a linear form with Re $a_{i}>0$, $\operatorname{Re}\left(\delta+\sum_{i=1}^{r} a_{i}\right)>0$. Then the zeta function $Z(L, \alpha, s)$ defined by

$$
Z(L, \alpha, s)=\sum_{n_{1}=1}^{\infty} \ldots \sum_{n_{r}=1}^{\infty} n_{1}^{\alpha_{1}} \ldots n_{r}^{\alpha_{r}}\left(a_{1} n_{1}+\ldots+a_{r} n_{r}+\delta\right)^{-s}, \quad \text { Res }>r+|\alpha|,
$$


has an analytic continuation in the whole complex plane, which is analytic except for simple poles at $s=1,2, \ldots, r+|\alpha|$. Furthermore

$$
Z(L, \alpha, 0)=\sum_{\left\{j_{1}, \ldots, j_{p}\right\}} J^{r-p}\left[\int_{\Delta\left(X_{j_{1}}, \ldots, X_{j_{p}}\right)} X^{\alpha} d X_{j_{1}} \ldots d X_{j_{p}}\right]
$$

where $\left\{j_{1}, \ldots, j_{p}\right\}$ ranges over all subsets of $\{1,2, \ldots, r\}$ in the summation and $\Delta\left(X_{j_{1}}, \ldots, X_{j_{p}}\right)$ is the domain in $\mathbb{R}^{p}$ defined by $L(X) \geq 0, X_{j_{1}} \leq 0, \ldots, X_{j_{p}} \leq 0$ when the coefficients $a_{i}(i=1, \ldots, r)$ and $\delta$ are real numbers.

Proof. For Re $s>0$, we have

$$
\left(a_{1} n_{1}+\ldots+a_{r} n_{r}+\delta\right)^{-s} \Gamma(s)=\int_{0}^{\infty} t^{s-1} \exp \left\{-\left(a_{1} n_{1}+\ldots+a_{r} n_{r}+\delta\right) t\right\} d t
$$

Hence for $\operatorname{Re} s>r+|\alpha|$, we have

$$
\begin{aligned}
Z(L, \alpha, s) \Gamma(s) & =\sum_{n \in \mathbb{N}^{r}} n^{\alpha} \int_{0}^{\infty} t^{s-1} \exp \left\{-\left(a_{1} n_{1}+\ldots+a_{r} n_{r}+\delta\right) t\right\} d t . \\
& =\int_{0}^{\infty} t^{s-1} e^{-\delta t} \prod_{i=1}^{r}\left(\sum_{n=1}^{\infty} n^{\alpha_{i}} e^{-a_{i} n t}\right) d t .
\end{aligned}
$$

It follows from Proposition 1 that

$Z(L, \alpha, 0)=$ the constant term in the expansion at $t=0$

$$
\text { of the function } F(t)=e^{-\delta t} \prod_{i=1}^{r}\left(\sum_{n=1}^{\infty} n^{\alpha_{i}} e^{-a_{i} n t}\right) \text {. }
$$

Note that

$$
\sum_{n=1}^{\infty} n^{\alpha_{i}} e^{-a_{i} n t}=\frac{\alpha_{i} !}{\left(a_{i} t\right)^{\alpha_{i}+1}}+\sum_{n \geq \alpha_{i}+1} \frac{(-1)^{\alpha_{i}} B_{n}\left(a_{i} t\right)^{n-\alpha_{i}-1}}{n \cdot\left(n-\alpha_{i}-1\right) !} \quad(i=1, \ldots, r) .
$$

Thus the constant in $F(t)$ is a sum of products of the form

$$
\prod_{i=1}^{p} \frac{\alpha_{i} !}{\left(a_{i}\right)^{\alpha_{i}+1}} \sum_{|\beta|=\alpha_{1}+\ldots+\alpha_{p}+p} \prod_{i=p+1}^{r} \frac{(-1)^{\alpha_{i}} B_{\beta_{i}+\alpha_{i}+1} a_{i}^{\beta_{i}}}{\left(\beta_{i}+\alpha_{i}+1\right) \cdot \beta_{i} !} \cdot \frac{(-1)^{\beta_{r+1}} \delta^{\beta_{r+1}}}{\beta_{r+1} !}
$$

after a permutation in the index set $\{1,2, \ldots, r\}$. Here $|\beta|=\beta_{p+1}+\ldots+\beta_{r}+\beta_{r+1}$.

On the other hand, an elementary calculation shows that

$$
\begin{aligned}
& \int_{\Delta\left(X_{1}, \ldots, X_{p}\right)} X^{\alpha} d X_{1} \ldots d X_{p} \\
& \quad=\left[\prod_{i=1}^{p} \frac{\alpha_{i} !}{\left(a_{i}\right)^{\alpha_{i}+1}}\right] \frac{\prod_{i=p+1}^{r} X_{i}^{\alpha_{i}}\left[-\left(a_{p+1} X_{p+1}+\ldots+a_{r} X_{r}+\delta\right)\right]^{\alpha_{1}+\ldots+\alpha_{p}+p}}{\left(\alpha_{1}+\ldots+\alpha_{p}+p\right) !} .
\end{aligned}
$$

Thus the one-to-one correspondence between the constant terms in the product $F(t)$ and polynomials arising from integrations of $X^{\alpha}$ over various simplexes is clear. Hence our assertion follows.

As an immediate consequence of our theorem and Proposition 2, we have the following formula for the special values of $Z(P, \beta, s)=Z\left(\prod_{j=1}^{n} L_{j}, \beta, s\right)$. 
Corollary 1 (Eie [6]). The special value of $Z(P, \beta, s)$ at nonpositive integers $-m$ is given by

$$
Z(P, \beta,-m)=\frac{1}{n} \sum_{j=1}^{n} \sum_{\left\{j_{1}, \ldots, j_{p}\right\}} J^{r-p}\left[\int_{\Delta_{j}\left(X_{j_{1}}, \ldots, X_{j_{p}}\right)} X^{\beta} P^{m}(X) d X_{j_{1}} \ldots d X_{j_{p}}\right]
$$

Here in the second summation, $\left\{j_{1}, \ldots, j_{p}\right\}$ ranges over all subsets of $\{1, \ldots, r\}$. The domain of integration $\Delta_{j}\left(X_{j_{1}}, \ldots, X_{j_{p}}\right)$ is defined by $\left(X_{j_{1}}, \ldots, X_{j_{p}}\right) \in \mathbb{R}^{p}$, $L_{j}(X) \geq 0, X_{j_{1}} \leq 0, \ldots, \ldots, X_{j_{p}} \leq 0$, when the coefficients of $L_{j}(X)$ are positive real numbers.

The exact same procedure can be used to evaluate the special values of the zeta function $\zeta(A, x, s)$ considered by Shintani in [9] in order to determine the special values of Dedekind zeta functions of totally real number fields. Shintani's formulas for the special values of $\zeta(A, x, s)$ were improved by the first author in the present form below [7]. For any $r \times n$ matrix $A=\left[a_{j i}\right]$ with positive entries and any $r$-tuple of complex numbers $x=\left(x_{1}, \ldots, x_{r}\right)$. The zeta function $\zeta(A, x, s)$ is defined as

$$
\zeta(A, x, s)=\sum_{n_{1}=0}^{\infty} \ldots \sum_{n_{r}=0}^{\infty} \prod_{j=1}^{n}\left[a_{j 1}\left(n_{1}+x_{1}\right)+\ldots+a_{j r}\left(n_{r}+x_{r}\right)\right]^{-s}, \quad \operatorname{Re} s>\frac{r}{n} .
$$

Let $B_{n}(x)$ be the Bernoulli polynomial defined by $B_{n}(x)=\sum_{k=0}^{n}\left(\begin{array}{l}n \\ k\end{array}\right) B_{n-k} x^{k}$.

For any subset $S$ of $I=\{1,2, \ldots, r\}$ and polynomial $g(u)$ with variables in $\left\{u_{i} \mid i \in S\right\}$ and $g(u)=\sum_{|\alpha|=0}^{p} b_{\alpha} \prod_{i \in S} u_{i}^{\alpha_{i}}$, we let

$$
J_{S}[g]=\sum_{|\alpha|=0}^{p} b_{\alpha} \cdot \prod_{i \in S} \frac{-B_{\alpha_{i}+1}\left(x_{i}\right)}{\alpha_{i}+1}
$$

if $g(u)=\sum_{|\alpha|=0}^{p} b_{\alpha} \prod_{i \in S} u_{i}^{\alpha_{i}}$. When $S=\phi$, we let $J_{\phi}[c]=c$ for any constant $c$.

Then Shintani's formula for $\zeta(A, x, 1-m)$ can be improved by the following.

Corollary 2 (Shintani $[8]$ ). For any positive integer $m$,

$$
\zeta(A, x, 1-m)=\frac{1}{n} \sum_{S} J_{S}\left[\sum_{j=1}^{n} \int_{\Delta_{j, S}(u)} P^{m-1}(u) \prod_{j \notin S} d u_{j}\right] .
$$

Here $S$ ranges over all non-empty subsets of $I=\{1,2, \ldots, r\}$,

$$
P(u)=\prod_{j=1}^{n}\left[a_{j 1} u_{1}+\ldots+a_{j r} u_{r}\right]
$$

and $\Delta_{j, S}(u)$ is the simplex defined by $\Delta_{j, S}(u):\left(u_{j}\right)_{j \notin S}, u_{j} \leq 0, L_{j}(u)=a_{j 1} u_{1}+$ $\ldots+a_{j r} u_{r} \geq 0$. 


\section{A FURTHER IDENTITY}

Let $\alpha, \beta>0$ with $\alpha \beta=\pi^{2}$ and $n$ be a positive integer. Then the following identity is well known ([1], page 276):

$$
\begin{aligned}
& 2^{2 n} \sum_{k=0}^{n+1} \frac{B_{2 n+2-2 k}}{(2 n+2-2 k) !} \frac{B_{2 k}}{(2 k) !} \alpha^{n+1-k}(-\beta)^{k} \\
& =-\alpha^{-n}\left\{\frac{1}{2} \zeta(2 n+1)+\sum_{k=1}^{\infty} \frac{k^{-2 n-1}}{e^{2 \alpha k}-1}\right\}+(-\beta)^{-n}\left\{\frac{1}{2} \zeta(2 n+1)+\sum_{k=1}^{\infty} \frac{k^{-2 n-1}}{e^{2 \beta k}-1}\right\}
\end{aligned}
$$

Here we extend it to an identity of Bernoulli polynomials.

Proposition 3. For positive numbers $\alpha, \beta$ with $\alpha \beta=\pi^{2}$ and real numbers $u, v$ such that $0 \leq u, v \leq 1$, we have

(a)

$$
\begin{aligned}
2^{2 n} \sum_{k=0}^{n+1} \frac{B_{2 k}(v)}{(2 k) !} \frac{B_{2 n-2 k+2}(u)}{(2 n-2 k+2) !} \alpha^{n-k+1}(-\beta)^{k} \\
=-\frac{1}{2} \alpha^{-n} \sum_{k=1}^{\infty} \frac{k^{-2 n-1} \cos (2 k \pi v)\left[e^{2 k u \alpha}+e^{2 k(1-u) \alpha}\right]}{e^{2 k \alpha}-1} \\
\quad+\frac{1}{2}(-\beta)^{-n} \sum_{k=1}^{\infty} \frac{k^{-2 n-1} \cos (2 k u \pi)\left[e^{2 k(1-v) \beta}+e^{2 k v \beta}\right]}{e^{2 k \beta}-1} ;
\end{aligned}
$$

(b)

$$
\begin{aligned}
& 2^{2 n} \sum_{k=0}^{n} \frac{B_{2 k+1}(v)}{(2 k+1) !} \frac{B_{2 n-2 k+1}(u)}{(2 n-2 k+1) !} \alpha^{n-k+1 / 2} \beta^{k+1 / 2}(-1)^{k} \\
&=-\frac{1}{2} \alpha^{-n} \sum_{k=1}^{\infty} \frac{k^{-2 n-1} \sin (2 k \pi v)\left[e^{2 k u \alpha}-e^{2 k(1-u) \alpha}\right]}{e^{2 k \alpha}-1} \\
&+\frac{1}{2}(-\beta)^{-n} \sum_{k=1}^{\infty} \frac{k^{-2 n-1} \sin (2 k \pi u)\left[e^{2 k(1-v) \beta}-e^{2 k v \beta}\right]}{e^{2 k \beta}-1} .
\end{aligned}
$$

Proof. For the time being, we suppose $u, v>0$. For any given $\epsilon>0$, consider the zeta function

$$
Z_{\epsilon}(s)=\sum_{n_{1}=0}^{\infty} \sum_{n_{2}=0}^{\infty}\left[\sqrt{\alpha}\left(n_{1}+u\right)+(\epsilon+i \sqrt{\beta})\left(n_{2}+v\right)\right]^{-s}, \quad \operatorname{Re} s>2 .
$$

$Z_{\epsilon}(s)$ has its analytic continuation and its special value at $s=-2 n$ is

$$
Z_{\epsilon}(-2 n)=\frac{(2 n) !}{\sqrt{\alpha}(\epsilon+i \sqrt{\beta})} \sum_{k=0}^{2 n+2} \frac{B_{k}(v)}{k !} \frac{B_{2 n-k+2}(u)}{(2 n-k+2) !}(\sqrt{\alpha})^{2 n-k+2}(\epsilon+i \sqrt{\beta})^{k}
$$


by Corollary 2. On the other hand, we set

$$
\begin{aligned}
F_{\epsilon}(t) & =\sum_{n_{1}=0}^{\infty} \sum_{n_{2}=0}^{\infty} \exp \left\{-\left[\sqrt{\alpha}\left(n_{1}+u\right)+(\epsilon+i \sqrt{\beta})\left(n_{2}+v\right) t\right]\right\} \\
& =\frac{\exp [-(\sqrt{\alpha} u+\epsilon v+i \sqrt{\beta} v) t]}{\left(1-e^{-\sqrt{\alpha}}\right)\left(1-e^{-(\epsilon+i \sqrt{\beta}) t}\right)}, \\
F(t) & =\frac{\exp [-(\sqrt{\alpha} u+i \sqrt{\beta} v) t]}{\left(1-e^{-\sqrt{\alpha} t}\right)\left(1-e^{-i \sqrt{\beta} t}\right)} .
\end{aligned}
$$

By Proposition 1, we have

$$
Z_{\epsilon}(-2 n)=(2 n) ! \times \text { the coefficient of } t^{2 n} \text { in the asymptotic expansion }
$$

$$
\text { at } t=0 \text { of the function } F_{\epsilon}(t) \text {. }
$$

It follows that for any $\epsilon>0$, we have the identity

$$
\begin{aligned}
& \frac{(2 n) !}{\sqrt{\alpha}(\epsilon+i \sqrt{\beta})} \sum_{k=0}^{2 n+2} \frac{B_{k}(v)}{k !} \frac{B_{2 n-k+2}(u)}{(2 n-k+2) !}(\sqrt{\alpha})^{2 n-k+2}(\epsilon+i \sqrt{\beta})^{k} \\
= & (2 n) ! \times \text { the coefficient of } t^{2 n} \text { in the asymptotic expansion }
\end{aligned}
$$

$$
\text { at } t=0 \text { of the function } F_{\epsilon}(t) \text {. }
$$

As $\epsilon$ approaches 0 , both sides in the formula above converge to nice limits, respectively. Thus we have

$$
\begin{aligned}
& \frac{1}{\sqrt{\alpha \beta} i} \sum_{k=0}^{2 n+2} \frac{B_{k}(v)}{k !} \frac{B_{2 n-k+2}(u)}{(2 n-k+2) !}(\sqrt{\alpha})^{2 n-k+2}(i \sqrt{\beta})^{k} \\
= & \text { the coefficient of } t^{2 n} \text { in the asymptotic expansion } \\
= & \frac{1}{2 \pi i} \int_{|z|=\delta} z^{-2 n-1} F(z) d z
\end{aligned}
$$

where $\delta$ is a positive number such that $\delta<\min (2 \sqrt{\alpha}, 2 \sqrt{\beta})$ and the direction on the circle $|z|=\delta$ is counterclockwise. By the residue theorem, the contour integral is equal to

$$
\begin{aligned}
& -\sum_{k \in \mathbb{Z}, k \neq 0}\left\{\text { Residue of } z^{-2 n-1} F(z) \text { at } z=\frac{2 k \pi i}{\sqrt{\alpha}}, \frac{2 k \pi}{\sqrt{\beta}}\right\} \\
=- & \frac{1}{2^{2 n+1}} \cdot \frac{1}{\sqrt{\alpha \beta i}} \alpha^{-n} \sum_{k=1}^{\infty} \frac{k^{-2 n-1}\left(e^{-2 k \pi i v+2 k(1-u) \alpha}+e^{2 k \pi i v+2 k u \alpha}\right)}{e^{2 k \alpha}-1} \\
& +\frac{1}{2^{2 n+1}} \cdot \frac{1}{\sqrt{\alpha \beta} i}(-\beta)^{-n} \sum_{k=1}^{\infty} \frac{k^{-2 n-1}\left(e^{-2 k \pi u i+2 k \beta v}+e^{2 k \pi u i+2 k(1-v) \beta}\right)}{e^{2 k \beta}-1}
\end{aligned}
$$

Comparing the real and imaginary parts of the resulted formula, we get our assertion for $u, v>0$.

Note that both sides of the identities in (a) and (b) are analytic functions of $u$ and $v$, it holds for $u>0, v>0$, so it also holds for $u=0$ or $v=0$. 
Remark. When $n$ is a negative integer, we have

$$
\frac{1}{2 \pi i} \int_{|z|=\delta} z^{-2 n-1} F(z) d z=0
$$

if $n<-1$ since $z^{2} F(z)$ is analytic at $z=0$. It follows that for $0<u, v<1$ and $m>1$,

$$
\begin{aligned}
& \alpha^{m} \sum_{k=1}^{\infty} \frac{k^{2 m-1} \cos (2 k \pi v)\left[e^{2 k u \alpha}+e^{2 k(1-u) \alpha}\right]}{e^{2 k \alpha}-1} \\
= & (-\beta)^{m} \sum_{k=1}^{\infty} \frac{k^{2 m-1} \cos (2 k \pi u)\left[e^{2 k u \beta}+e^{2 k(1-v) \beta}\right]}{e^{2 k \beta}-1} .
\end{aligned}
$$

For the limit case $u=v=0$, we have

$$
\alpha^{m}\left[\zeta(1-2 m)+2 \sum_{k=1}^{\infty} \frac{k^{2 m-1}}{e^{2 k \alpha}-1}\right]=(-\beta)^{m}\left[\zeta(1-2 m)+2 \sum_{k=1}^{\infty} \frac{k^{2 m-1}}{e^{2 k \beta}-1}\right] .
$$

This is equivalent to

$$
\alpha^{m} \sum_{k=1}^{\infty} \frac{k^{2 m-1}}{e^{2 k \alpha}-1}-(-\beta)^{m} \sum_{k=1}^{\infty} \frac{k^{2 m-1}}{e^{2 k \beta}-1}=\left[\alpha^{m}-(-\beta)^{m}\right] \frac{B_{2 m}}{4 m}
$$

as given on page 261 in [1].

\section{REFERENCES}

1. Bruce C. Berndt, Ramanujan's Notebooks, Part II, Springer-Verlag, 1989. MR 90b:01039

2. P. Cassou-Nouguès, Valeurs aux entiers négatifs des functions zêta p-adiques, Invent. Math. 51 (1979), 25-59.

3. - Valeurs aux entiers négatifs des séries de Dirichlet associées à un polynôme I, J. Number Theory 14 (1982), 32-64. MR 83e: 12012

4. Séries de Dirichlet et intégrales associées à un polynôme a deux indéterminés, J. Number Theory 23 (1986), 1-54. MR 87j:11086

5. Minking Eie, On a Dirichlet series associated with a polynomial, Proc. Amer. Math. Soc. 110 (1990), 583-590. MR 91m:11071

6. - The special values at negative integers of Dirichlet series associated with polynomials of several variables, Proc. Amer. Math. Soc. 119 (1993), 51-61. MR 93k:11082

7. __ A note on Bernoulli numbers and Shintani generalized Bernoulli polynomial, Trans. Amer. Math. Soc. 348 (1996), 1117-1136. MR 96h:11011

8. I. M. Gelfand and G. E. Shilov, Generalized function, vol. 1, Academic Press, 1964. MR 55:8786a

9. T. Shintani, On evaluation of zeta functions of totally real algebraic number fields at nonpositive integers, J. Fac. Sci. Univ. Tokyo Sect. IA Math. 23 (1976), 393-417. MR 55:266

10. On the values at $s=1$ of certain L-functions of totally real algebraic number fields, Algebraic number theory, International Symposium, Kyoto, 1976. MR 56:11962

11. D. Zagier, Valeurs des fonctions zêta des corps quadratiques réels aux entiers négatifs, Astérisque (1977), 41-42. MR 52:10684

Institute of Applied Mathematics, National Chung Cheng University, Ming-Hsiung, Chia-Yi 621, Taiwan, Republic of China

E-mail address: mkeie@math.ccu.edu.tw 